\title{
Luminescence properties of magnetic polarons in EuTe: Theoretical description and experiments in magnetic fields up to $28 \mathrm{~T}$
}

\author{
A. B. Henriques, F. C. D. Moraes, and G. D. Galgano \\ Instituto de Física, Universidade de São Paulo, Caixa Postal 66318, CEP 05315-970 São Paulo, Brazil
}

A. J. Meaney, P. C. M. Christianen, and J. C. Maan

High Field Magnet Laboratory, Institute for Molecules and Materials, Radboud University Nijmegen, NL-6525 ED Nijmegen, The Netherlands

E. Abramof and P. H. O. Rappl

LAS-INPE, 12227-010 São José dos Campos, Brazil

(Received 29 June 2014; published 9 October 2014)

\begin{abstract}
The recent discovery of a polaron-associated zero phonon line in the band-edge photoluminescence of high optical quality EuTe crystals opens up the prospect of answering long-standing questions about the polaron internal structure, thermal stability, and generation efficiency. Here, a Schrödinger equation for the polaron was formulated and resolved by using both variational and self-consistent methods. The theory is in good agreement with measurements of the zero phonon line as a function of magnetic field and temperature, and it could be applied to other polaronic systems. It is deduced that, in EuTe, at $0 \mathrm{~K}$, a polaron carries a magnetic moment of $610 \mu_{B}$, and its binding energy is $27 \mathrm{meV}$. However, this binding energy does not carry the usual meaning of thermal stability, because it decreases drastically when the sample is warmed up. For instance, at $T=100 \mathrm{~K}$, the binding energy is already reduced to only $6 \mathrm{meV}$. The thermal destruction of a polaron is brought about by thermal fluctuations of the spin lattice that suppress the electron's self-energy. Photoluminescence excitation spectra of EuTe demonstrate that the photogeneration of polarons becomes increasingly inefficient when the energy of the pumping photon is increased above the band gap.
\end{abstract}

DOI: 10.1103/PhysRevB.90.165202

PACS number(s): 78.55.-m, 78.20.Ls, 71.38.-k

\section{INTRODUCTION}

Europium telluride (EuTe) is an old-fashionable magnetic semiconductor that has been investigated for many decades [1-4]. The intrinsic source of magnetism in EuTe and other europium chalcogenides is the large spin $(S=7 / 2)$ of the $\mathrm{Eu}$ atom, which occupies all lattice sites of the crystal. Additionally, EuTe is optically transparent in the red and infrared wavelength ranges. The combination of huge magnetism and optical transparency in a single material attracts device engineers still today $[5,6]$, but EuTe already raised technological interest nearly half a century ago [7], when it became commercially available. However, technological interest waned when it was discovered that EuTe is an antiferromagnet with a very low Néel temperature $\left(T_{\mathrm{N}}=9.6 \mathrm{~K}\right)$, which is impractical for device applications. Nevertheless, EuTe and other Eu chalcogenides remain ideal magnetic systems that can be used to test the principles of operation of spin-related devices [8], and to investigate many-body physical phenomena, e.g., spin waves [9] and magnetic polarons [2,4,10-14].

Despite the long research history, only recently it was demonstrated how light can be used to induce a magnetic moment in EuTe, through the photocreation of magnetic polarons [15]. In Ref. [15], a narrow PL emission associated with a pure electronic transition was discovered, a so-called zero phonon line (ZPL). Is was demonstrated that the photoexcited electron that produces the ZPL is dressed by a halo of canted lattice spins, forming a magnetic polaron [16]. The experimental demonstration of magnetic polarons was done by using EuTe epitaxial layers of very high chemical purity and few structural defects, which became available in the last decade when the modern molecular beam epitaxy (MBE) technique began to be employed to grow EuTe crystals [17,18]. The superior quality of EuTe grown by MBE is demonstrated by its improved optical properties, e.g., new band-edge absorption of unprecedent sharpness [17], narrow photoluminescence (PL) lines at higher energies than ever seen before [18], novel second-harmonic generation [19], and a rich spectrum of magneto-refractive effects previously unknown [20]. Based on these discoveries, it became possible to successfully describe the EuTe band-edge electronic structure in the framework of one-electron band theory $[21,22]$, whose validity in rare-earth oxides was questioned in the past [23] due to divergences between theory and the experimental data available at the time [24]. MBE can also be used to integrate Eu chalcogenides into a silicon substrate and other III-V semiconductor structures [25,26], which enhances the prospects of using EuTe in novel optospintronic devices.

The above-mentioned technical and scientific advances, and above all the discovery of a polaron-related ZPL, open the prospect of answering questions such as what is the internal spin structure of the newly discovered polarons, how well may they be modelled theoretically, how can polarons be generated efficiently, what is the polaron binding energy, and how high in temperature may the control of magnetic order, seen in EuTe at helium temperatures [15], be taken? These are long-standing fundamental questions; the answer to them is under debate or even missing, despite the many of investigations already done. The present investigation answers these questions on the basis of a theoretical model and its confrontation with the results of magneto-optical experiments on high-quality epitaxial layers of EuTe, grown by the molecular beam epitaxy technique. The theoretical model and its predictions are described in Sec. II, technical experimental details are given in Sec. III, 
experimental results and comparison with theory are given in Sec. IV, and conclusion presented in Sec. V.

\section{MAGNETIC POLARON THEORY}

\section{A. Polaron Hamiltonian}

If an electron is promoted from the valence band into the conduction band through photon absorption in EuTe, prior to radiative recombination the electron will relax into the lowest possible state (dubbed " $X$ state") $[15,18,27]$. The exchange interaction between the photoexcited electron in an $X$ state and the lattice spins (the $X f$ interaction) favors alignment of all spins in a common direction and can be described by an effective magnetic field $B_{X f} \sim 1 \mathrm{~T}$, which acts on the lattice spins in the neighborhood of the photoexcited electron [15]. This effective magnetic field opposes the exchange interaction between the lattice spins (the $f f$ interaction), which is antiferromagnetic [28]. If the $X f$ interaction overpowers the $f f$ one, then a magnetic polaron may be formed, which is when the photoexcited electron breaks the natural antiferromagnetic alignment in its vicinity, generating a local nonzero magnetic moment. Magnetic polarons may also arise due to electrons bound to impurities and defects, and it has been suggested that they may be exploited to raise the critical temperature in rare-earth ferromagnets $[13,14]$.

To describe the localized photoexcited electron, the envelope-wave-function approach [29] is employed. In an isotropic crystal such as EuTe, the photoexcited electron is described by a spherically symmetric envelope wave function $\psi(r)$, where $r$ is the distance to the center of the polaron.

The total energy of a photoexcited electron surrounded by a cloud of canted lattice spins (measured in respect to the energy of a free Bloch electron) is given by the sum

$$
E_{\text {Pol }}=\mathcal{K}+U_{\text {Coul }}+E_{X f}+E_{f f}+E_{Z},
$$

where $\mathcal{K}$ represents the kinetic of the photoexcited electron, $U_{\text {Coul }}$ is its Coulomb energy in the field of the photoexcited hole,

$$
E_{X f}=-J_{X f} S \sum_{i} \frac{a^{3}}{4}\left|\psi\left(R_{i}\right)\right|^{2} \sin \phi\left(R_{i}\right)
$$

is the $X f$ energy $[17,30]\left[J_{X f}\right.$ is the constant for the exchange interaction between the photoexcited electron and the lattice spins [15], $S=\frac{7}{2}$ is the spin of an Eu atom, $a$ is the EuTe lattice parameter, $R_{i}$ is the distance from the $i$ th lattice spin to the center of the polaron, $\phi\left(R_{i}\right)$ is the canting angle of the $i$ th lattice spin, as shown in Fig. 1],

$$
E_{f f}=\sum_{i} 12\left|J_{1}+J_{2}\right| S^{2}\left[\sin ^{2} \phi\left(R_{i}\right)-b^{2}\right]
$$

is the change in the lattice ff energy induced by the photoexcited electron [ $J_{1}$ and $J_{2}$ are the first- and second-neighbor ff exchange constants, respectively, $b=\frac{B}{B_{\mathrm{SAT}}}$ is the value of $\sin \phi\left(R_{i}\right)$ in the absence of the photoexcited electron, $B \leqslant$ $B_{\mathrm{SAT}}$ is the absolute value of the applied magnetic field, $B_{\mathrm{SAT}}$ is the minimum external magnetic field required to impose complete ferromagnetic order in the unperturbed lattice [31] ],

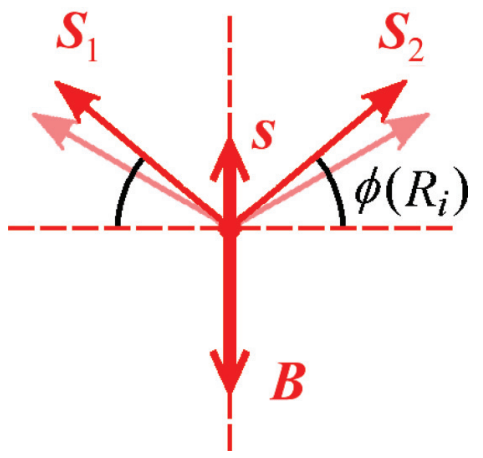

FIG. 1. (Color online) The two antiferromagnetic sublattices are indicated by vectors $S_{1}$ and $S_{2}$, under the effect of the resultant magnetic field, i.e., the superposition of an external field and the exchange field of the photoexcited electron, indicated by vector $s$. The lighter arrows indicate the sublattice spin orientation in the absence of a photoexcited electron. The magnetic field acting on the spins is assumed to be larger than the spin-flop field, and the photoexcited electron is relaxed. At a given distance, $R_{i}$, to the polaron center, both sublattices are tilted by the same angle $\phi$ with respect to their unperturbed equilibrium orientation indicated by the horizontal dashed line.

and

$$
E_{Z}=-g_{S} \mu_{\mathrm{B}} S B \sum_{i}\left[\sin \phi\left(R_{i}\right)-b\right]
$$

is the change in the Zeeman energy of the lattice spins, induced by the photoexcited electron $\left(g_{S}=2\right.$ is the gyromagnetic factor for an $\mathrm{Eu}$ spin in the lattice and $\mu_{\mathrm{B}}$ is Bohr's magneton). The Zeeman energy of the photoexcited electron itself, in the range of fields considered here, is much smaller than all other energies and will be ignored. It is assumed that the effective magnetic field acting on the lattice spins is larger than the spin-flop field $B_{\mathrm{SF}} \sim 0.08 \mathrm{~T}$ [32], hence the lattice spins belonging to different sublattices are oriented symmetrically to the effective field [15], as shown in Fig. 1. It is also assumed that the spin of the photoexcited electron is relaxed [15,27], meaning that the effective field $B_{X f}$ points in the direction of the applied magnetic field, if one is present.

Last but not least, the canting angle is allowed to vary continuously, which would be strictly valid only when $S \rightarrow \infty$ but is a suitable approximation in the present case, considering the large spin $S=7 / 2$ of the Eu atoms [11].

Due to the spherical symmetry, $\phi\left(R_{i}\right)$ is constant for a given radius $R_{i}$, and by minimizing $E_{f f}+E_{X f}+E_{Z}$ in respect to $\sin \phi\left(R_{i}\right)$, we find

$$
\sin \phi\left(R_{i}\right)= \begin{cases}1 & \text { if } A\left|\psi\left(R_{i}\right)\right|^{2}+b>1 \\ A\left|\psi\left(R_{i}\right)\right|^{2}+b & \text { otherwise, }\end{cases}
$$

where

$$
A=\frac{J_{X f}}{96\left|J_{1}+J_{2}\right| S} a^{3} .
$$

By taking advantage of the continuum approximation [3], whereby the discrete distribution of Eu spins in the crystal lattice is replaced by a continuous one, with a density of spins equal to $4 / a^{3}$ characteristic of the face-centered cubic lattice, the polaron problem can be formulated in terms of a very 
succinct Schrödinger equation. One writes

$$
E_{f f}+E_{X f}+E_{Z}=\int_{0}^{\infty} 4 \pi r^{2} d r|\psi(r)|^{2} V_{\mathrm{Pol}}(r),
$$

and

$$
E_{\mathrm{Pol}}=\int_{0}^{\infty} 4 \pi r^{2} d r \psi^{*}(r) \mathcal{H}_{\mathrm{Pol}} \psi(r),
$$

where the Hamiltonian operator is given by

$$
\mathcal{H}_{\mathrm{Pol}}=-\frac{\hbar^{2}}{2 m^{*} r^{2}} \frac{d}{d r}\left(r^{2} \frac{d}{d r}\right)-\frac{k e^{2}}{\varepsilon r}+V_{\mathrm{Pol}}(r) .
$$

The first term in Eq. (8) is the kinetic energy operator $\left(m^{*}\right.$ is the effective mass of the photoexcited electron), the second term is the Coulomb interaction operator between the electron and the photoexcited hole, which is screened by a dielectric constant $\varepsilon$, and $V_{\mathrm{Pol}}(r)$ is the self-energy term, which unites the $f f, X f$, and Zeeman energies [Eqs. (3), (2), and (4), respectively] in a single compact and concise expression:

$$
V_{\mathrm{Pol}}(r)=-J_{X f} S \begin{cases}1-\frac{(1-b)^{2}}{2 A|\psi(r)|^{2}}, & r \leqslant r_{\mathrm{c}} \\ \frac{1}{2} A|\psi(r)|^{2}+b & \text { otherwise, }\end{cases}
$$

where $r_{\mathrm{c}}$ is the radius of the spherical ferromagnetic core at the center of the polaron [i.e., the region where $\phi(r) \equiv \pi / 2$; $r_{\mathrm{c}}$ is determined by the condition $A\left|\psi\left(r_{\mathrm{c}}\right)\right|^{2}+b=1$ or $r_{\mathrm{c}}=0$ if this condition is never satisfied].

\section{B. Ground state of the polaron}

The ground state of the polaron is given by the minimum energy solution of the Schrödinger equation

$$
\mathcal{H}_{\text {Pol }} \psi(r)=E_{\text {Pol }} \psi(r) .
$$

The Schrödinger equation must be solved by using the self-consistent method [33] because the confining potential $V_{\mathrm{Pol}}(\mathrm{r})$ is dependent on the wave function, as Eq. (9) shows. Alternatively, an approximate solution can be found by using the variational method. Although less accurate, the variational method has the advantage of producing a fully analytical solution. In this paper both solutions are presented and compared. To the best of the authors knowledge, this is the first time that the self-consistent method has been used to tackle the polaron problem.

The first known theoretical treatment of magnetic polarons in EuTe assumed a variational wave function equal to a constant within the polaron sphere and zero outside of it $[2,34]$. A better choice is Bohr's function, which will be used here, as done by other authors describing impurity-stabilized polarons [10,11] and photoinduced ones [35],

$$
\psi\left(r, a_{\mathrm{B}}\right)=\frac{e^{-r / a_{\mathrm{B}}}}{\sqrt{\pi a_{\mathrm{B}}^{3}}} .
$$

The effective Bohr radius $a_{\mathrm{B}}$ is the variational parameter. With this choice the radius of the ferromagnetic core, defined in Eq. (9), can be expressed analytically:

$$
r_{\mathrm{c}}= \begin{cases}\frac{a_{\mathrm{B}}}{2} \ln \frac{A}{(1-b) \pi a_{\mathrm{B}}^{3}} & \text { if } A>(1-b) \pi a_{\mathrm{B}}^{3} \\ 0 & \text { otherwise. }\end{cases}
$$

TABLE I. EuTe parameters used in the theoretical modeling of the polaron.

\begin{tabular}{lcccccccc}
\hline \hline Parameter & $J_{1}(\mathrm{~K})$ & $J_{2}(\mathrm{~K})$ & $a(\AA)$ & $B_{\mathrm{SF}}(\mathrm{T})$ & $m^{*}$ & $\varepsilon$ & $J_{X f}(\mathrm{meV})$ \\
\hline Value & 0.043 & -0.15 & 6.6 & 0.08 & $0.3 m_{0}$ & 8.3 & 83 \\
Reference & {$[1]$} & {$[1]$} & {$[36]$} & {$[32]$} & {$[37]$} & {$[18,38]$} & This work \\
\hline \hline
\end{tabular}

The entry parameters used in all calculations are given in Table I. For the parameters given in Table I, no bound state is produced if the Coulomb term of the Hamiltonian is set to zero, in agreement with Ref. [11], which concluded theoretically that free conduction band electrons cannot form magnetic polarons in bulk EuTe.

Figures 2 and 3 show the results of the calculations obtained both by the self-consistent and variational methods. The variational results were obtained by substituting Eq. (11) in Eq. (7) and minimizing the energy obtained, $E_{\mathrm{Pol}}$, to yield the best variational parameter $a_{\mathrm{B}}$. However, the analytical expression obtained for $E_{\mathrm{Pol}}$ by using the variational method is too large to be given here, and therefore only the final values will be given. For $B=0, a_{\mathrm{B}}=1.27 a$ is found. Figure 2(a) shows the polaron part of the confining potential, $V_{\mathrm{Pol}}(r)$, and the radial probability distribution associated with the envelope wave
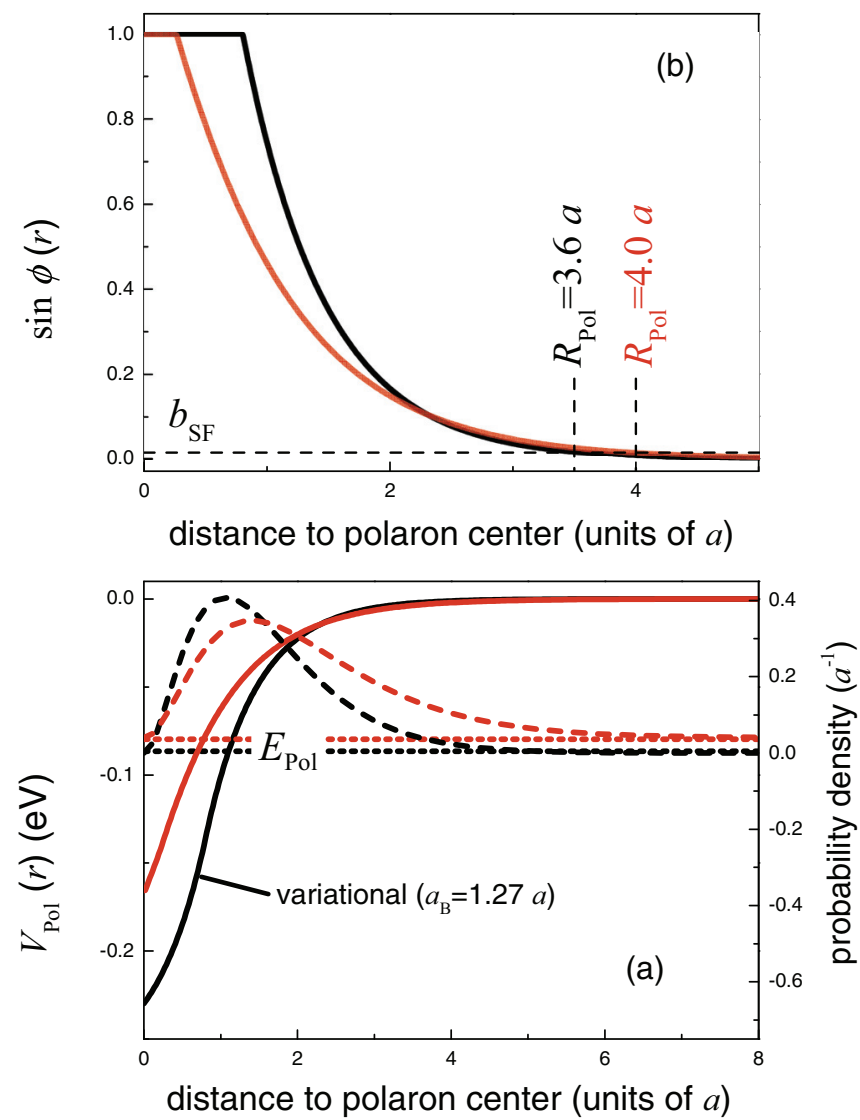

FIG. 2. (Color online) Polaron ground state obtained by the selfconsistent method (lighter curves) and the variational method (darker curves) at $B=0$. (a) $V_{\mathrm{Pol}}(r)$ (solid line), probability density (dashed line), and energy of the ground state (dotted line). (b) Radial dependence of the canting angle. 


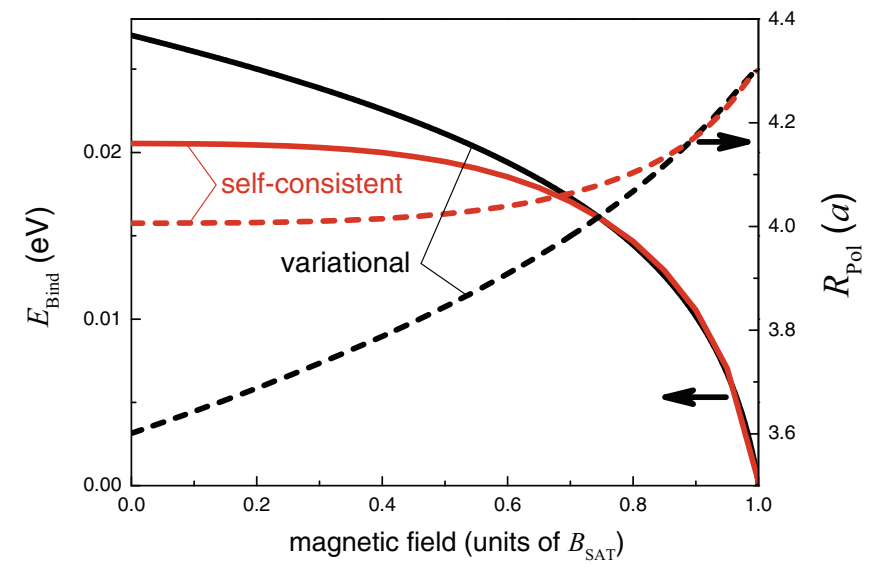

FIG. 3. (Color online) Magnetic-field dependence of the polaron binding energy (solid lines) and of the polaron radius (dashed lines) obtained by the self-consistent (lighter curves) and the variational methods (darker curves).

function of the confined electron for $B=0$. The self-consistent $V_{\mathrm{Pol}}(r)$ is less confining than the variational one given by Eq. (9), and the self-consistent orbital is more extended. The energy of the photoexcited electron bound state is found to be $-0.0942 \mathrm{eV}$ for the variational method, and $-0.0860 \mathrm{eV}$ for the self-consistent one. The difference between these values is explained by the fact that the variational function is only approximately consistent with Eq. (10), in which the confining potential itself [Eq. (9)] depends on the wave function.

Figure 2(b) shows the sine of the canting angle as a function of distance to the polaron center. The variational calculation produces a ferromagnetic core of radius $r_{\mathrm{c}}=0.95 a$, whereas the self-consistent result is $r_{\mathrm{c}}=0.47 a$. Given that the nearestneighbor distance in the face-centered cubic EuTe lattice is $0.71 a$, then a small ferromagnetic core is predicted by the variational model, but no ferromagnetic core is expected in the self-consistent approximation, which characterizes the polaron as type I [11].

In the idealized picture above the photoexcited electron is allowed to polarize all lattice spins, no matter how distant they are from the polaron center. In reality, the polarization action of the photoexcited electron is limited to the region where the effective magnetic field generated by the photoexcited electron wins the competition with other sources of effective magnetic fields; for instance, the magnetic anisotropy [39], which is described by a spin-flop field of $B_{\mathrm{SF}} \sim 0.1 \mathrm{~T}$ [32]. Consequently the polaron radius will be finite. The radius of the polaron can be estimated by taking the spin-flop field as a measure of the minimum effective field that the photoexcited electron must generate in order to have any effect on the lattice polarization. Then the polarization action of the photoexcited electron on the lattice spins, which is measured by how much the photoelectron changes the (sine of the) canting angle, which from Eq. (5) is given by $A|\psi(r)|^{2}$, must be greater than the change in $\sin \phi$ that would be caused by a magnetic field of magnitude $B_{\mathrm{SF}}$, which is simply [15] $b_{\mathrm{SF}}=B_{\mathrm{SF}} / B_{\mathrm{SAT}}$. Thus the polaron volume is defined by the values of $r$ that satisfy the inequality

$$
A|\psi(r)|^{2} \geqslant b_{\mathrm{SF}}
$$

The equality occurs at $r=R_{\text {Pol }}$, which by using Eq. (11) produces an analytical expression for the polaron radius,

$$
R_{\mathrm{Pol}}=\frac{a_{\mathrm{B}}}{2} \ln \frac{A}{b_{\mathrm{SF}} \pi a_{\mathrm{B}}^{3}},
$$

if the variational approximation is used, which gives $R_{\mathrm{Pol}}=$ 3.6 $a$ for $B=0$. In contrast, in the self-consistent approximation, $R_{\mathrm{Pol}}=4.0 \mathrm{a}$ is found numerically from the equality condition in Eq. (13).

The average canting angle of the lattice spins within the polaron will be given by

$$
\langle\sin \phi\rangle=\Omega_{\mathrm{Pol}}^{-1} \int_{0}^{R_{\mathrm{Pol}}} 4 \pi r^{2} d r \sin \phi(r),
$$

where $\Omega_{\mathrm{Pol}}=\frac{4}{3} \pi R_{\mathrm{Pol}}^{3}$ is the polaron volume, which gives $\langle\phi\rangle=7^{\circ}$ for the variational model and $\langle\phi\rangle=5^{\circ}$ for the self-consistent one. Both the theoretically estimated $R_{\text {Pol }}$ and the average canting angle $\langle\phi\rangle$ are in approximate agreement with the experimental estimates given in Ref. [15], where the polaron radius was found to be $3.2 a$, and the complementary angle to $\phi$ was found to be $\sim 80^{\circ}$.

From Eq. (15) the total magnetic moment of a polaron can be estimated to be

$$
\mathcal{M}_{\mathrm{Pol}}=\Omega_{\mathrm{Pol}} M_{\mathrm{SAT}}\langle\sin \phi\rangle,
$$

where $M_{\mathrm{SAT}}=\frac{4}{a^{3}} g_{S} \mu_{\mathrm{B}} S$ is the saturation magnetization of the crystal, which gives $\mathcal{M}_{\mathrm{Pol}}=650 \mu_{\mathrm{B}}$ and $\mathcal{M}_{\mathrm{Pol}}=610 \mu_{\mathrm{B}}$ in the variational and self-consistent calculations, respectively.

When the applied magnetic-field intensity reaches $B_{\mathrm{SAT}}$, the lattice spins attain complete ferromagnetic alignment, and the polaron potential becomes a constant, $V_{\mathrm{Pol}}(r)=-J_{X f} S$, as Eq. (9) shows. In this limit, the variational Bohr function becomes an exact solution of the polaron Hamiltonian. Indeed, the variational and self-consistent calculations converge to a single result as $b \rightarrow 1$, as Fig. 3 shows. However, the selfconsistent result, which is an exact solution of the polaron Schrödinger equation, produces a polaron radius that is almost independent of the magnetic field and varies by at most $\sim 5 \%$, whereas the variational result for $R_{\mathrm{Pol}}$ varies linearly with $B$, and can be increased by as much as $20 \%$ by the application of a magnetic field.

The binding energy of the polaron, $E_{\mathrm{Bind}}$, is equal to the photoexcited electron's self-energy, i.e., the energy it gains by polarizing the surrounding lattice spins. Put in another way, $E_{\text {Bind }}$ is the difference between the total binding energy of the photoexcited electron and its binding energy when the lattice spins are unpolarized. The former is the difference between the energy of the polaron state, $E_{\text {Pol }}$, given by Eq. (10), and the continuum threshold, which according to Eq. (9) is equal to $-b J_{X f} S$; the latter is just the effective Rydberg, $R^{*}=m^{*} k^{2} e^{4} /\left(2 \varepsilon^{2} \hbar^{2}\right)$. Thus, as illustrated in Fig. 4,

$$
E_{\text {Bind }}=-b J_{X f} S-E_{\text {Pol }}-R^{*} .
$$

The calculated $E_{\text {Bind }}$, using Eq. (17), is shown in Fig. 3 as a function of the applied magnetic field.

As already mentioned, the $X f$ interaction can be described by an effective magnetic field. From Eq. (2) this effective magnetic field, at a distance $r$ from the polaron center, is 


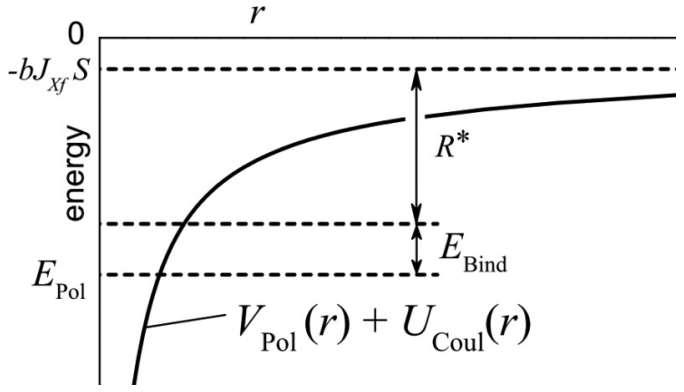

FIG. 4. Confining potential, fundamental energy level, and parameters that define the polaron binding energy.

easily deduced to be

$$
B_{X f}(r)=\frac{J_{X f}}{g_{S} \mu_{B}} \frac{a^{3}}{4}|\psi(r)|^{2},
$$

whose average value within the polaron volume is

$$
\left\langle B_{X f}\right\rangle=\frac{1}{\Omega_{\mathrm{Pol}}} \frac{J_{X f}}{g_{S} \mu_{B}} \frac{a^{3}}{4} .
$$

The effective $X f$ field adds to the magnetic field applied externally, therefore the required external field required to impose ferromagnetic alignment within a polaron is lower by $\left\langle B_{X f}\right\rangle$ than in the rest of the crystal. By using the parameters given in Table I and $R_{\mathrm{Pol}} \sim 4 a,\left\langle B_{X f}\right\rangle \sim 1 \mathrm{~T}$ is obtained, in approximate agreement with the experimental value [15].

Based on the above theory, valid for $T=0 \mathrm{~K}$, predictions can be made for polaron effects in the high-temperature limit, $T \gg T_{\mathrm{N}}$, when the EuTe system approaches paramagnetic behavior. In this limit, $E_{f f} \rightarrow 0$ and $E_{X f}$ dominates the selfenergy of the photoexcited electron for $B=0$ (i.e., when $E_{Z}=0$ ). In the continuum approximation, Eq. (2) can be rewritten as

$$
E_{X f}=-J_{X f} S\langle\sin \phi\rangle ;
$$

on the other hand, the average magnetization inside the polaron is given by $\langle M\rangle=M_{\mathrm{SAT}}\langle\sin \phi\rangle$, hence

$$
\langle\sin \phi\rangle=\langle m\rangle,
$$

where $\langle m\rangle=\langle M\rangle / M_{\mathrm{SAT}}$ is the reduced magnetization (the magnetization measured in units of the maximum-possible value, $\left.M_{\mathrm{SAT}}\right)$. But in the paramagnetic limit, $\langle m\rangle$ approaches the Brillouin function, i.e.,

$$
\lim _{T \gg T_{\mathrm{N}}}\langle m\rangle=B_{S}(T, B),
$$

where $S=7 / 2$ for EuTe. Furthermore, within a polaron we may approximate $B$ by $\left\langle B_{X f}\right\rangle$, the average effective magnetic field acting on the lattice spins contained in the polaron volume. Substituting Eqs. (21) and (22) into Eq. (20), we get

$$
\lim _{T \gg T_{\mathrm{N}}} E_{X f}=-J_{X f} S B_{S}\left(T,\left\langle B_{X f}\right\rangle\right)
$$

The binding energy of the polaron is simply the absolute value of the self-energy, i.e.,

$$
\lim _{T \gg T_{\mathrm{N}}} E_{\mathrm{Bind}}=J_{X f} S B_{S}\left(T,\left\langle B_{X f}\right\rangle\right)
$$

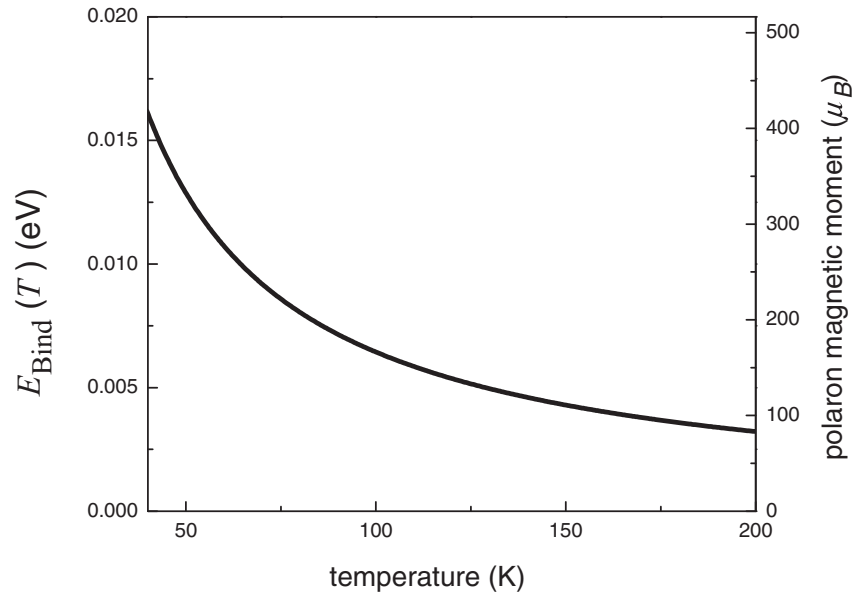

FIG. 5. Calculated magnetic polaron binding energy (left scale) and magnetic moment (right scale) as a function of temperature, using Eqs. (24) and (25), respectively.

Finally, the magnetic moment of a polaron is given by $\mathcal{M}_{\mathrm{Pol}}=\Omega_{\mathrm{Pol}} M_{\mathrm{SAT}}\langle m\rangle$, and can also be estimated in the high-temperature limit by substituting Eqs. (21) and (22) into Eq. (16), which gives

$$
\lim _{T \gg T_{\mathrm{N}}} \mathcal{M}_{\mathrm{Pol}}=\Omega_{\mathrm{Pol}} M_{\mathrm{SAT}} B_{S}\left(T,\left\langle B_{X f}\right\rangle\right) .
$$

The calculated binding energy, using Eq. (24), is shown in Fig. 5. It can be seen that the binding energy plummets towards room temperature. This is explained by the increasing thermal agitation of the lattice spins when the sample is heated, which suppresses the ability of the photoexcited electron to polarize the lattice spins around itself, thereby inhibiting polaron formation. At $T=100 \mathrm{~K}$, the binding energy is $6 \mathrm{meV}$ (only a small fraction of the $T=0 \mathrm{~K}$ value), which is approximately equal to $k_{B} T$, hence polaron formation is unlikely already at $T \sim 100 \mathrm{~K}$. Parallel to the fast decrease of the binding energy when the temperature is raised, Eq. (25) shows that the magnetic moment of a polaron also decreases in the same proportion. For instance, at $T=100 \mathrm{~K}$, the magnetic moment of a polaron is reduced to only $\mathcal{M}_{\mathrm{Pol}}=150 \mu_{\mathrm{B}}$.

\section{EXPERIMENT}

The near-gap photoluminescence (PL) and photoluminescence excitation (PLE) in EuTe were measured by using very low excitation powers (below $\sim 100 \mu \mathrm{W} / \mathrm{cm}^{2}$ ), which is mandatory for resolving the ZPL [15]. The samples were grown by molecular beam epitaxy on $\mathrm{BaF}_{2}$ substrates [36]. The thickness of the epitaxial layers was in the range 1-4.2 $\mu \mathrm{m}$. In the PL investigation, the excitation source was a Nd:YAG $532 \mathrm{~nm}$ laser, and for PLE a Xenon lamp coupled to a single-grating $0.25 \mathrm{~m}$ monochromator was used. Light was conveyed to the sample and collected from the sample by using optical fibers and in situ optics assembled in a homemade sample holder, similar to the one described in Ref. [40]. The sample holder was used to position the sample inside a cryostat in complete isolation from ambient light sources. When the PL intensity is very low (as is the case here, because extremely low excitation levels were employed), our light collection 
method, instead of using a conventional cryostat with windows and freestanding optical elements, is advantageous for two reasons (for a detailed description see Ref. [41]): (1) the in situ optics allows us to collect light over a greater solid angle; (2) ambient light sources are completely excluded, whereas they hinder measurements in an open-lens-optics setup. In the PLE experiment, the excitation light was left-hand or right-hand circularly polarized by using a circular polarizer contained in the in situ optics. All measurements were taken at a temperature of $T=5 \mathrm{~K}$, under the effect of a magnetic field of up to $28 \mathrm{~T}$ and applied in the Faraday geometry. Measurements taken in fields above $10 \mathrm{~T}$ where done at the High Magnetic Field Laboratory of Radboud University in Nijmegen (HMFL).

\section{RESULTS AND DISCUSSION}

Measured PL spectra are shown as a function of the applied magnetic field in Fig. 6(a), whereby a weak manifold of equally spaced lines could be resolved. As an example, the black solid line in Fig. 6(b) shows the PL for $B=7 \mathrm{~T}$, in which seven lines, equally spaced by the EuTe optical phonon energy [42] of $17.5 \mathrm{meV}$, are well resolved. The manifold intensity fits well with a Poisson distribution (for details see Ref. [15]), characteristic of a radiative transition between two electronic states but coupled to a single phonon mode $[43,44]$. Therefore, the whole of the PL band is ascribed to a single photoexcited electronic state, the so-called $X$ state, as detailed in Ref. [15]. The light dashed curves in Fig. 6(b) show the isolated individual phonon replica from which the position, width, and intensity of the ZPL can be extracted.

In contrast to the commonly observed PL in EuTe reported by other authors, which consists of the unresolved superposition of several lines, the ZPL is associated with a single and pure electronic transition. Therefore, the ZPL displacement, when the crystal is submitted to a perturbation of some kind, measures directly the displacement of the electronic energy levels. This is not the case for a PL line that englobes several electronic transitions or multiphonon emissions, because in the latter case the energy shift of the PL maximum may be driven by an uneven change in the oscillator strengths of the transitions comprising the PL band and not by any real shift of energy levels. Moreover, since the ZPL is associated with a polaron state, an energy shift of the ZPL is equal to a change in the energy of the polaron. Figure 7 shows the shift in the position of the maximum of the ZPL as a function of magnetic field. Below the saturation field of $B_{\mathrm{SAT}}=7.3$ $\mathrm{T}$ (the intensity of the applied magnetic field required to impose ferromagnetic spin alignment within a polaron), the linear redshift is described by a slope of $38 \mathrm{meV} / \mathrm{T}$. For comparison, the shift in the polaron energy, $E_{\mathrm{Pol}}$, predicted by the self-consistent calculation using parameters given in Table I, is also shown in Fig. 7(a). The theory predicts the correct behavior and, up to the saturation field, the agreement between theory and experiment is slightly better if the value of the parameter $J_{X f}$ is taken to be $J_{X f}=83 \mathrm{meV}$, as given in Table I, instead of $J_{X f}=77 \mathrm{meV}$, estimated in an earlier work [15].

At $B=B_{\mathrm{SAT}}=7.3 \mathrm{~T}$ (i.e., when $b=1.0$ ), full ferromagnetic alignment within the polaron is achieved. However,
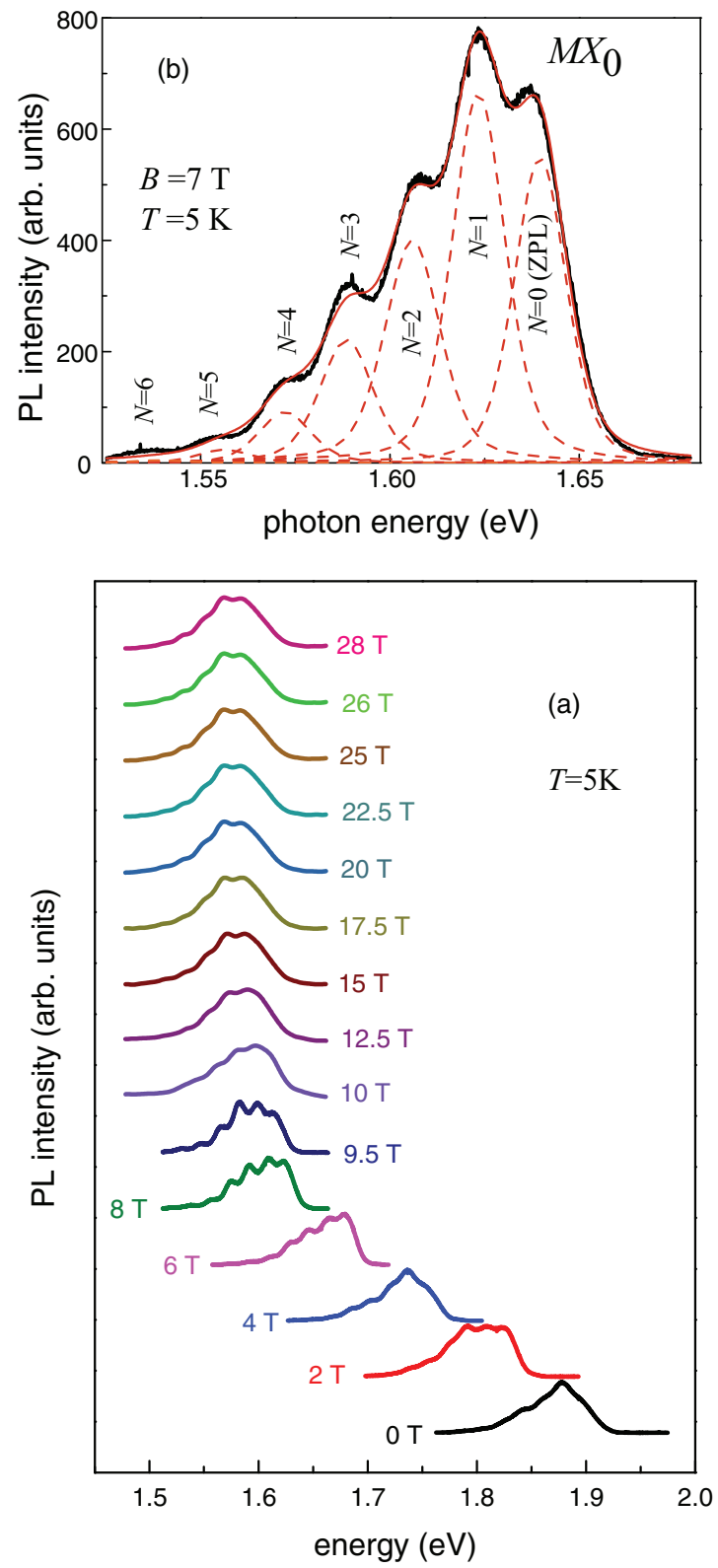

FIG. 6. (Color online) (a) Normalized PL spectra for an epitaxial sample of thickness $4.2 \mu \mathrm{m}$ as a function of field. For clarity, the curves were displaced vertically. The magnetic field corresponding to each curve is indicated. Above $B=10 \mathrm{~T}$ the spectra were taken using a slightly larger excitation power, which leads to a less-well-resolved phonon structure [15]. (b) PL spectrum at $B=7 \mathrm{~T}$. Black line is the experimental result, the light curve depicts a fit with a Poisson distribution of lines [15], from which the ZPL is identified. Dashed curves show the individual phonon lines that compose the fit.

at this field the lattice spins outside the polaron sphere are not yet saturated. Increasing the field up to $B=8.3 \mathrm{~T}$, then ferromagnetic alignment is imposed everywhere, the magnetic polaron ceases to exist, and the position of the ZPL is expected to remain constant. However, Fig. 7 shows that the ZPL continues to redshift (by about 15 to $20 \mathrm{meV}$ ) even when the applied magnetic field is greater than the saturation field, up to $15 \mathrm{~T}$, where the redshift comes to a halt. The small redshift between 8.3 and $15 \mathrm{~T}$ is probably associated with a 


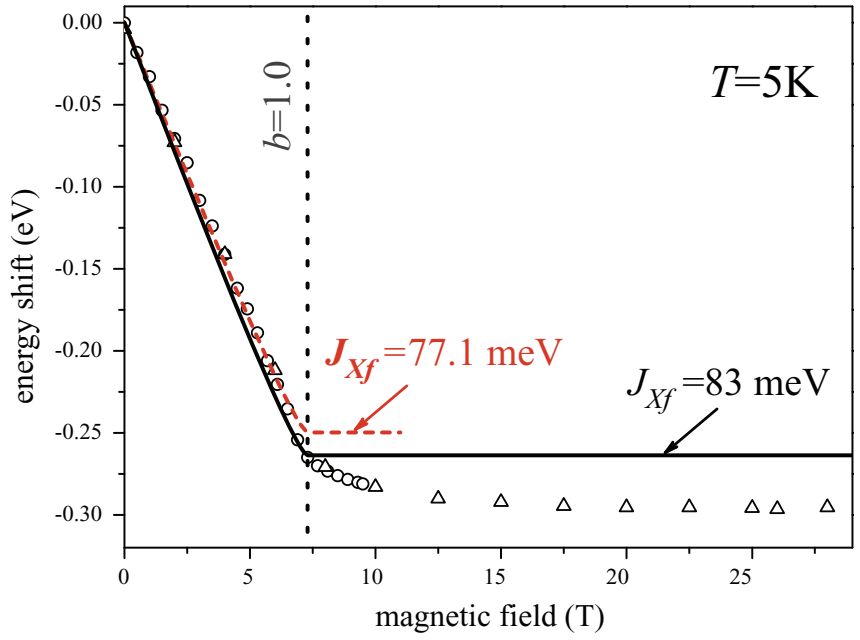

FIG. 7. (Color online) Redshift of the ZPL as a function of applied magnetic field (lower scale). The upper scale shows the field in units of the saturation field $B_{\mathrm{SAT}}$ required to align the lattice spins. Circles (triangles) represent data taken on an EuTe epitaxial layer of $1.0 \mu \mathrm{m}(4.2 \mu \mathrm{m})$. Theory is shown for $J_{X f}=77 \mathrm{meV}$ and $J_{X f}=83 \mathrm{meV}$.

magnetic-field suppression of roughness in the confining potential of the photoexcited electron, associated with structural or chemical inhomogeneities.

The position of the ZPL as a function of temperature at $B=0$ was already reported (Fig. 4 in Ref. [15]). The ZPL could be followed up to a temperature of about $30 \mathrm{~K}$. Above $30 \mathrm{~K}$, the PL becomes too weak, and the ZPL can no longer be resolved. Nevertheless, between the Néel temperature and $30 \mathrm{~K}$, a blueshift of about $20 \mathrm{meV}$ is observed, which is typical of the optical-band-edge absorption spectrum of EuTe in a magnetic field [1]. When an external magnetic field is present, the blueshift is associated with increasing lattice spin fluctuations, which reduce the magnetization induced by the magnetic field, which in turn reduces the $X f$ energy gain of the photoexcited electron. However, the ZPL blueshift observed here occurs when no external magnetic field exists, which provides independent proof that the photoexcited electron generates an effective magnetic field. The blueshift also indicates that increasing the temperature progressively destroys the polaron, as expected from the theoretical model (see end of Sec. II B).

Finally, the photoluminescence excitation spectrum of the ZPL is shown in Fig. 8 as a function of the applied magnetic field. The PLE threshold is characterized by the same quadratic redshift seen in the optical absorption of EuTe, which is due to the narrowing of the band gap [45]. However, at any applied field, the PLE spectrum was always independent of the polarization of the excitation light, in striking contrast to the optical absorption spectrum, which shows a huge circular dichroism [45]. This result can be interpreted in the following way: Although at the band-edge threshold the optical densities are different for left- and right-hand circularly polarized photons, both photons end up being absorbed anyway, because the samples used here are very thick, thus both photons generate photoexcited electrons, albeit in different excited electronic states. However, prior to

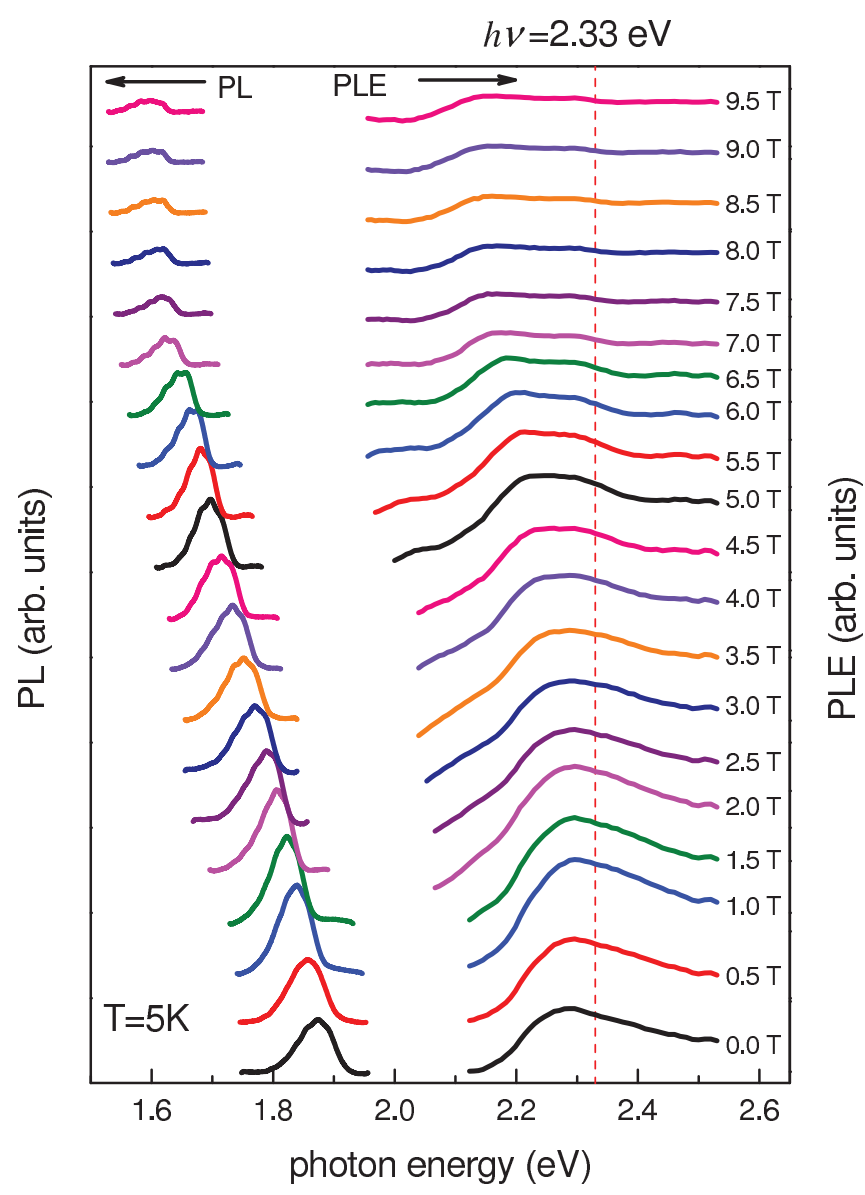

FIG. 8. (Color online) PL (left scale) and PLE (right scale) as a function of the applied magnetic field. PL spectra shown were measured by using an excitation tuned to the maximum of the PLE spectrum.

recombination, the photoexcited electron relaxes energy and spin [27], loosing memory of the excited electronic state it occupied initially. Thus the PL produced is uncorrelated with the polarization of the excitation light.

Additionally, when the energy of the excitation photons is driven above the EuTe band gap, the PLE intensity decreases, which is again in sharp contrast to the optical absorption, which steeply increases $[24,45]$. The decreasing PLE signal points to a decreasing connection between energy levels higher in the conduction band and the relaxed state that originates the ZPL. This suggests that electrons excited to states of higher energy have an increasing chance of encountering a nonradiative recombination path, which is quite plausible. Another aspect worthy of notice is the decreasing PLE signal with increasing magnetic field at a fixed energy just above the band gap. This is the case for the PL with photons of energy $532 \mathrm{~nm}(2.33 \mathrm{eV})$, which is near the maximum of the PLE spectrum at $B=0$, as the dotted line in Fig. 8 shows. Upon increasing the applied magnetic field, the EuTe band gap hugely redshifts, hence the $2.33 \mathrm{eV}$ excitation photons are strongly moved out of resonance, and for a fixed excitation power the PL signal decreases. Therefore the observation of a decreasing PL signal with increasing magnetic field can be partly explained by the increasing disconnection between the 
hot photogenerated electrons and the relaxed photoexcited state, and not necessarily by a change in the symmetry of the relaxed photoexcited state, as has been suggested in the literature [18].

\section{CONCLUSIONS}

In summary, the magnetic polaron in EuTe has been revisited in high-quality crystals grown by $\mathrm{MBE}$ and thoroughly investigated both theoretically and experimentally. A very concise single-particle effective Hamiltonian operator, describing the polaron in a magnetic field, was formulated, and its Schrödinger equation was solved exactly by using the self-consistent method for the first time. Although in general the self-consistent method produces very similar results to those obtained by the variational method, some results are distinctly different, e.g., the size of the ferromagnetic core, the size of the polaron itself, and the magnetic-field dependence of the latter. The theory correctly predicts the ZPL shift in a magnetic field, and with increasing temperature of the sample, demonstrating its reliability. The theory is very compact and depends on few entry parameters (exchange constants, effective mass, and dielectric constant), and could be used to describe magnetic polarons not only in other europium chalcogenides, but in many other materials as well.
Confrontation of the theory with the experimental data gathered on EuTe provides an estimate for the $X f$ exchange constant, $J_{X f}=83 \mathrm{meV}$. At $T \rightarrow 0 \mathrm{~K}$, the polaron radius is $4 a$, the binding energy of the polaron is $27 \mathrm{meV}$, and its magnetic moment is $610 \mu_{\mathrm{B}}$. However, the polaron binding energy and its magnetic moment decrease drastically when temperature is increased and, by $T \sim 100 \mathrm{~K}$, the polaron is effectively destroyed. This is explained by the increasing agitation of the lattice spins when the temperature is raised, which makes them less susceptible to the effective magnetic field the photoexcited electron produces.

The PLE intensity is independent on the polarization of the excitation light, which demonstrates that the spin of the photoexcited electron that generates the magnetic polaron is completely relaxed when the polaron is formed. Finally, it was demonstrated that polaron generation is most efficient when the excitation-light photons are in resonance with the EuTe energy gap and progressively decreases when the excitationphoton energy is increased beyond the EuTe gap.

\section{ACKNOWLEDGMENTS}

This work was supported by the Brazilian agencies CNPq (Project 304685/2010-0) and FAPESP (Project 2012/23406$0)$. We acknowledge the support of HFML-RU/FOM, member of the European Magnetic Field Laboratory (EMFL). Part of this work has been supported by EuroMagNET II under the EU contract number 228043.
[1] P. Wachter, Handbook on the Physics and Chemistry of Rare Earths (North-Holland, Amsterdam, 1979), Vol. 1.

[2] T. Kasuya, A. Yanase, and T. Takeda, Solid State Commun. 8, 1543 (1970).

[3] A. Mauger and C. Godart, Phys. Rep. 141, 52 (1986).

[4] E. L. Nagaev, J. Magn. Magn. Mater. 110, 39 (1992).

[5] J. Philip, A. Punnose, B. I. Kom, K. M. Reddy, S. Layne, J. O. Holmes, B. Satpati, P. R. Leclair, T. S. Santos, and J. S. Moodera, Nat. Mater. 5, 298 (2006).

[6] A. H. MacDonald, P. Schifer, and N. Samarth, Nat. Mater. 4, 195 (2005).

[7] J. O. Dimmock, C. E. Hurwitz, and T. B. Reed, J. Appl. Phys. 40, 1336 (1969).

[8] Q. I. Yang, J. Zhao, L. Zhang, M. Dolev, A. D. Fried, A. F. Marshall, S. H. Risbud, and A. Kapitulnik, Appl. Phys. Lett. 104, 082402 (2014).

[9] S. O. Demokritov, N. M. Kreines, and V. I. Kudinov, Sov. Phys. JETP 65, 369 (1987).

[10] A. Mauger and D. L. Mills, Phys. Rev. Lett. 53, 1594 (1984).

[11] A. Mauger and D. L. Mills, Phys. Rev. B 31, 8024 (1985).

[12] I. Zutic, J. Fabian, and S. Das Sarma, Rev. Mod. Phys. 76, 323 (2004).

[13] P. Liu and J. Tang, Phys. Rev. B 85, 224417 (2012).

[14] F. Natali, B. J. Ruck, H. J. Trodahl, D. L. Binh, S. Vezian, B. Damilano, Y. Cordier, F. Semond, and C. Meyer, Phys. Rev. B 87, 035202 (2013).

[15] A. B. Henriques, G. D. Galgano, E. Abramof, B. Diaz, and P. H. O. Rappl, Appl. Phys. Lett. 99, 091906 (2011).
[16] D. R. Yakovlev and W. Ossau, Introduction to the Physics of Diluted Magnetic Semiconductors, Springer Series in Materials Science, Vol. 144 (Springer, Berlin, 2010), pp. 221-262.

[17] L. K. Hanamoto, A. B. Henriques, N. F. Oliveira, P. H. O. Rappl, E. Abramof, and A. Y. Ueta, J. Phys.: Condens. Matter 16, 5597 (2004).

[18] W. Heiss, R. Kirchschlager, G. Springholz, Z. Chen, M. Debnath, and Y. Oka, Phys. Rev. B 70, 035209 (2004).

[19] B. Kaminski, M. Lafrentz, R. V. Pisarev, D. R. Yakovlev, V. V. Pavlov, V. A. Lukoshkin, A. B. Henriques, G. Springholz, G. Bauer, E. Abramof et al., Phys. Rev. Lett. 103, 057203 (2009).

[20] R. R. Subkhangulov, A. B. Henriques, P. H. O. Rappl, E. Abramof, T. Rasing, and A. V. Kimel, Sci. Rep. 4, 4368 (2014).

[21] A. B. Henriques, M. A. Manfrini, P. H. O. Rappl, and E. Abramof, Phys. Rev. B 77, 035204 (2008).

[22] A. B. Henriques, E. Abramof, and P. H. O. Rappl, Phys. Rev. B 80, 245206 (2009).

[23] G. Guntherodt, Eur. Phys. J. B 18, 37 (1974).

[24] G. Guntherodt, P. Wachter, and D. M. Imboden, Physik der kondensierten Materie 12, 292 (1971).

[25] A. Schmehl et al., Nat. Mater. 6, 882 (2007).

[26] J. Lettieri, V. Vaithyanathan, S. K. Eah, J. Stephens, V. Sih, D. D. Awschalom, J. Levy, and D. G. Schlom, Appl. Phys. Lett. 83, 975 (2003).

[27] F. G. G. Hernandez, A. B. Henriques, P. H. O. Rappl, and E. Abramof, Int. J. Mod. Phys. B 23, 2999 (2009). 
[28] J. S. Smart, Effective Field Theories of Magnetism (W. B. Saunders Company, Philadelphia \& London, 1966).

[29] G. Bastard, Wave Mechanics Applied to Semiconductor Heterostructures (Les Éditions de Physique, Les Ulis, 1988).

[30] Here the $X f$ interaction energy for a photoexcited electron in an extended state, given in Ref. [17], whereby it interacts equally with all lattice spins, was generalized to a localized electron state, assuming that the $X f$ interaction with a given lattice spin is proportional to the probability that the photoexcited electron is found in the volume of the crystal that corresponds to that particular lattice spin.

[31] J. Feinleib and C. R. Pidgeon, Phys. Rev. Lett. 23, 1391 (1969).

[32] J. W. Battles and G. E. Everett, Phys. Rev. B 1, 3021 (1970).

[33] L. D. Landau and E. M. Lifshitz, Quantum Mechanics, NonRelativistic Theory, Course of Theoretical Physics (AddisonWesley, Reading, 1965), Vol 3, Chap. 10.

[34] M. Umehara and T. Kasuya, J. Phys. Soc. Jpn. 40, 340 (1976).

[35] M. Umehara, Phys. Rev. B 52, 8140 (1995).
[36] B. Díaz, E. Granado, E. Abramof, P. H. O. Rappl, V. A. Chitta, and A. B. Henriques, Phys. Rev. B 78, 134423 (2008).

[37] J. Vitins and P. Wachter, Phys. Rev B 12, 3829 (1975).

[38] S. Kück and H. Werheit, EuTe: Crystal Structure, Physical Properties, Landolt-Börnstein - Group III Condensed Matter, Vol. III/41D (Springer, Berlin, 2000).

[39] S. Blundell, Magnetism in Condensed Matter (Oxford University Press, New York, 2001), p. 95.

[40] A. B. Henriques, G. D. Galgano, B. L. Diaz, P. H. O. Rappl, and E. Abramof, J. Phys.: Condens. Matter 19, 406234 (2007).

[41] R. F. Oliveira, Ph.D. thesis, University of São Paulo, 2005, available for download at http://www.teses.usp.br/teses/disponiveis/ 43/43134/tde-06032014-144125/.

[42] G. D. Holah, J. S. Webb, R. B. Dennis, and C. R. Pidgeon, Solid State Commun. 13, 209 (1973).

[43] T. H. Keil, Phys. Rev. 140, A601 (1965).

[44] A. B. Henriques and J. P. von der Weid, Solid State Commun. 56, 571 (1985).

[45] A. B. Henriques, A. Wierts, M. A. Manfrini, G. Springholz, P. H. O. Rappl, E. Abramof, and A. Y. Ueta, Phys. Rev. B 72, 155337 (2005). 